\title{
A Study on Improvement of RANS Analysis for Erosion of Density Stratified Layer of Multicomponent Gas by Buoyant Jet in a Containment Vessel
}

\author{
Satoshi Abe, Masahiro Ishigaki, Yasuteru Sibamoto and Taisuke Yonomoto \\ Thermalhydraulic Safety Research Group, Nuclear Safety Research Center, Japan Atomic Energy Agency, Ibaraki 319-1195, Japan
}

Received: March 19, 2015 / Accepted: May 06, 2015 / Published: July 31, 2015.

\begin{abstract}
The analysis on a density stratification layer consisting of multiple gases in the reactor containment vessel is important for the safety assessment of sever accidents. The JAEA (Japan Atomic Energy Agency) has started the project on the containment thermal hydraulics. We carried out CFD (computational fluid dynamics) analyses in order to investigate the erosion of the density stratification layer by a vertical buoyant jet under this project. We used the RANS (Reynolds averaged numerical simulation) and LES (large eddy simulation) models to analyze the erosion of a density stratification layer by a vertical buoyant jet in a small vessel which represents a containment vessel. This numerical study calculates the turbulent mixing of a two-component (air and helium) gas mixture. The turbulence models used for the RANS analyses are two types of $k$ - $\varepsilon$ models. The first model is the low Reynolds number $k-\varepsilon$ model developed by Launder and Sharma. The second model is modified from the first model in order to accurately consider the turbulent production and damping in a stratification layer. The results indicated while the erosion rate calculated by the low-Re $k$ - $\varepsilon$ model was much faster than that of the LES model, the modified $k-\varepsilon$ model could calculate the erosion rate similar to the LES result.
\end{abstract}

Key words: Density stratification layer, containment vessel, CFD, Kato-Launder modification, Katsuki model.

\section{Introduction}

Hydrogen detonation and combustion may arise in the reactor containment vessel during a severe accident, which may threaten the required containment functions. The Fukushima Dai-ichi nuclear power station accident in Japan in March 2011 has refocused attention on this issue. The gaseous behavior in the containment vessel is complicated in general because of its huge space and existence of multi gases having different densities.

The density stratified layer in the reactor containment vessel is important for the analyses on the hydrogen detonation and combustion.

CFD (computational fluid dynamics) analysis is one kind of computational tools for containment

Corresponding author: Satoshi Abe, Ph.D., research fields: computational fluid dynamics and turbulent transport phenomena. E-mail: abe.satoshi@jaea.go.jp. thermal-hydraulic analysis. Many activities in recent years were conducted to improve the performance of the CFD analyses. The OECD (Organization for Economic Co-operation and Development) international benchmark exercise ISP (International Standard Problem)-47 [1] was performed in order to assess the capabilities of both LP (lumped parameter) code and CFD codes in area of containment thermal hydraulics. The summary of ISP-47 concluded that, the results by CFD models were not generally better than those by LP code model, most CFD codes must be further improved regarding the modelling of condensation and the modelling of turbulence including the wall treatment.

In the OECD/SETH (SESAR (senior group of experts on nuclear safety research) thermal-hydraulic)-2 project [2], experiments were conducted with the PANDA and MISTRA facilities 
which are large scale simulated containment vessels for investigating containment thermal-hydraulic phenomena. Part of the project focuses on the erosion of the density stratification by vertical jet coming from below. Andreani, et al. [3] validated the GOTHIC code with the PANDA experimental results in the SETH-2 project. Their 2D analyses agreed well with the overall behavior observed in the experiment, although some disagreements were found in detailed behavior. This result indicated that, further improvement was necessary.

Based on those backgrounds, the JAEA (Japan Atomic Energy Agency) has started the ROSA-SA (rig of safety assessment-severe accident) project on the containment hydraulics [4]. This paper shows some results of this project, focusing on the transport phenomena for multicomponent gas in a containment vessel and introducing the modified turbulence model. A small scale rectangular vessel with $1.8 \mathrm{~m}$ in height, $1.5 \mathrm{~m}$ in length and $1.5 \mathrm{~m}$ in width is analyzed to investigate the erosion of the density stratification by vertical buoyant jet injected from the bottom. The RANS (Reynolds-Averaged Navier Stokes) and LES (large-eddy simulation) models are applied for the analysis. The RANS model was commonly used in ISP-47, among them $k-\varepsilon$ model [5] was mostly applied for analysis on density stratification erosion. It was found, however, that the RANS analyses by $k-\varepsilon$ model provided an overestimation on erosion [1]. Compared to this, the LES analysis can generally simulate not only mean-field but also filtered fluctuation field although it requires much more computational resource. In the present study, we applied two types of $k-\varepsilon$ models for the RANS analysis: the first model is the low-Reynolds type $k-\varepsilon$ model developed by Launder and Sharma [6], the second model is revised from the first model in order to accurately simulate the turbulent production and damping by use of the Kato-Launder modification [7] and the Katsuki model [8]. Those RANS analyses were compared with the LES analysis.

\section{RANS and LES Analyses Method}

\subsection{Government Equation and Turbulence Model}

The governing equations are continuity equation, momentum equation and transport equations of scalars (mass fraction and enthalpy) expressed as follows:

$$
\begin{gathered}
\frac{\partial \rho}{\partial t}+\frac{\partial \rho u_{i}}{\partial x_{i}}=0 \\
\frac{\partial \rho u_{i}}{\partial t}+\frac{\partial \rho u_{i} u_{j}}{\partial x_{j}}=-\frac{\partial p}{\partial x_{i}}+\frac{\partial}{\partial x_{j}}\left\{\mu\left(\frac{\partial u_{i}}{\partial x_{j}}+\frac{\partial u_{j}}{\partial x_{i}}\right)\right\} \\
\frac{\partial \rho Y_{k}}{\partial t}+\frac{\partial \rho u_{i} Y_{k}}{\partial x_{i}}=\frac{\partial}{\partial x_{i}}\left(\frac{\mu}{S c_{k}} \frac{\partial Y_{k}}{\partial x_{i}}\right) \\
\frac{\partial \rho h}{\partial t}+\frac{\partial \rho u_{i} h}{\partial x_{i}}=\frac{\partial}{\partial x_{i}}\left(\frac{\mu}{P r} \frac{\partial h}{\partial x_{i}}\right)+\frac{\partial p}{\partial t}
\end{gathered}
$$

where, $u_{i}, \rho, p, \mu, Y_{k}, S c_{k}, h$ and $\operatorname{Pr}$ are velocity in the $j$-th direction, density of fluid, pressure, viscosity coefficient, mass fraction of $k$-th gas, Schmidt number, enthalpy and Prandtl number, respectively. $F_{b}$ means buoyancy force term. The CFD analyses in this paper simulate the behavior of mixture of helium and air. The gasses are treated as perfect gas.

The LES and RANS analyses are performed, focusing on the erosion of density stratification by a vertical buoyant jet in the small vessel simulating the containment vessel. In the LES analyses, the filtering operation is applied to the governing equations in order to separate SGS (subgrid-scale) and GS (grid scale) components. Standard Smagorinsky type [9] for SGS modeling was employed for the LES analysis. The SGS stress $\left(\tau_{i j}\right)$ is derived with SGS eddy-viscosity coefficients as follows:

$$
\tau_{i j}-\frac{1}{3} \tau_{i i} \delta_{i j}=-\mu_{S G S}\left(\frac{\partial \widetilde{u}_{j}}{\partial x_{i}}+\frac{\partial \widetilde{u_{l}}}{\partial x_{j}}\right)
$$

where, the overbar and tilde mean an low-pass filter and density-weighted Favre filtered values, respectively. $\mu_{S G S}$ is SGS viscosity coefficient, which is derived with Eqs. (6) and (7):

$$
\begin{gathered}
\mu_{S G S}=\bar{\rho}\left(C_{S G S} f_{S} \Delta\right)^{2} \sqrt{2 \widetilde{S_{l j} \widetilde{S_{l \jmath}}}} \\
\widetilde{S_{l \jmath}}=\frac{1}{2}\left(\frac{\partial \widetilde{u_{J}}}{\partial x_{i}}+\frac{\partial \widetilde{u_{l}}}{\partial x_{j}}\right)
\end{gathered}
$$


where, $C_{S G S}$ is the Smagorinsky constant value and $f_{s}$ means the van Driest damping function, and $\Delta$ means the filter width of the low-pass filter. The SGS turbulent scalar flux (mass and enthalpy) is derived by the gradient diffusion hypothesis expressed as follows:

$$
\begin{gathered}
\bar{\rho}\left(\widetilde{u_{\imath} Y_{k}}-\widetilde{u_{l}} \cdot \widetilde{Y_{k}}\right)=-\frac{\mu_{S G S}}{S c_{k S G S}} \frac{\partial \widetilde{Y_{k}}}{\partial x_{i}} \\
\bar{\rho}\left(\widetilde{u_{\imath} h}-\widetilde{u_{l}} \cdot \tilde{h}\right)=-\frac{\mu_{S G S}}{P r_{S G S}} \frac{\partial \widetilde{h}}{\partial x_{i}}
\end{gathered}
$$

$S c_{k S G S}$ and $P r_{S G S}$ are SGS Schmidt number and Prandtl number, respectively.

The RANS analysis with the $k-\varepsilon$ model type represents the Reynolds-stress with eddy viscosity coefficients (Eq. (10)).

$$
\langle\rho\rangle\left[u_{i}^{\prime} u_{j}^{\prime}\right]=-\mu_{t}\left(\frac{\partial\left[u_{i}\right]}{\partial x_{j}}+\frac{\partial\left[u_{j}\right]}{\partial x_{i}}\right)+\frac{2}{3}\langle\rho\rangle k
$$

where, the prime mark means fluctuation component. The angle bracket \langle\rangle means the Reynolds averaged values, and the bracket [] means the density-weighted Favre averaged values. $\mu_{t}$ is turbulent viscosity coefficient, which is derived with turbulent kinetic energy and turbulent dissipation as follows:

$$
\mu_{t}=\langle\rho\rangle C_{\mu} f_{\mu} \frac{k^{2}}{\varepsilon}
$$

where, $C_{\mu}$ is constant value and $f_{\mu}$ means damping function. The turbulent scalar fluxes (mass fraction and enthalpy) are derived by the gradient diffusion hypothesis as follows:

$$
\begin{gathered}
\langle\rho\rangle\left[u_{i}^{\prime} Y_{k}^{\prime}\right]=-\frac{\mu_{t}}{S c_{k t}} \frac{\partial\left[Y_{k}\right]}{\partial x_{i}} \\
\langle\rho\rangle\left[u_{i}^{\prime} h^{\prime}\right]=-\frac{\mu_{t}}{P r_{t}} \frac{\partial[h]}{\partial x_{i}}
\end{gathered}
$$

where, $S c_{k t}$ and $P r_{t}$ are the turbulent Schmidt number and turbulent Prandtl number, respectively. The $k-\varepsilon$ modeling approach is widely used for industrial application due to its relatively low computational costs and generally good numerical stability. Eqs. (14) and (15) show transport equations for turbulent kinetic energy $(k)$ and turbulence dissipation $(\varepsilon)$, respectively.

$$
\begin{aligned}
& \frac{\partial\langle\rho\rangle k}{\partial t}+\frac{\partial\langle\rho\rangle\left[u_{i}\right] k}{\partial x_{i}}=P_{k}-\varepsilon+D i f f_{k} \\
& \frac{\partial\langle\rho\rangle \varepsilon}{\partial t}+\frac{\partial\langle\rho\rangle\left[u_{i}\right] \varepsilon}{\partial x_{i}}=P_{\varepsilon}-\Phi_{\varepsilon}+D i f f_{\varepsilon}
\end{aligned}
$$

where, $P_{k}$ and $P_{\varepsilon}$ mean the production terms for turbulent kinetic energy and turbulent dissipation, respectively. The third terms of RHS (right hand side) of Eqs. (14) and (15) represent diffusion terms.

\subsection{RANS Turbulence Model Modification Based on Low Re Type k-E Model}

In this study, two types of $k-\varepsilon$ models are employed in the RANS analyses. The first is the low Reynolds number $k$ - $\varepsilon$ type developed by Launder and Sharma [6]. The second is revised from the first model in order to accurately predict turbulent production and damping around density stratification. The modified model is relatively simple changing from the prototype model and has good numerical stability with consideration for applying to large scale test analyses. The following shows detail of the modified model.

In prototype of the $k-\varepsilon$ model, the production term of the turbulent kinetic energy $\left(P_{k}\right)$ in Eq. (14) is represented with turbulent viscosity $\left(\mu_{t}\right)$ and the mean rate of strain tensor $\left(S_{i j}\right)$ as shown in Eqs. (16) and (17):

$$
\begin{gathered}
P_{k}=\mu_{t} S S \\
S=\sqrt{2 S_{i j} S_{i j}}, S_{i j}=\frac{1}{2}\left(\frac{\partial\left[u_{i}\right]}{\partial x_{j}}+\frac{\partial\left[u_{j}\right]}{\partial x_{i}}\right)
\end{gathered}
$$

A stagnation point is formed by jet penetration into density stratification. It is generally understood that, the production of the turbulent kinetic energy is overestimated when using Eqs. (14) and (15). This may be one of the reasons for the overestimation of density stratification erosion by the RANS analyses as discussed in the previous study [1].

The Kato-Launder modification [7] is simple modification of the production term of the turbulent kinetic energy $\left(P_{k}\right)$ as follows:

$$
\begin{gathered}
P_{k}=\mu_{t} S \Omega \\
\Omega=\sqrt{2 \Omega_{i j} \Omega_{i j}}, \Omega_{i j}=\frac{1}{2}\left(\frac{\partial\left[u_{i}\right]}{\partial x_{j}}-\frac{\partial\left[u_{j}\right]}{\partial x_{i}}\right)
\end{gathered}
$$



Gas by Buoyant Jet in a Containment Vessel

where, $\Omega_{i j}$ is the mean rate of rotation tensor. In zones of irrotational flow such as around a stagnation point, $\Omega$ is nearly equal to 0 , which suppress the excessive turbulent kinetic energy production.

Turbulence damping in the density stratification is an important factor to simulate the stratification erosion phenomena [1]. In the present study, Katsuki model [8] is applied to the multicomponent flow analysis for better simulation of these turbulence phenomena.

The Reynolds-stress is described as follows based on concept of WET model [10], the wealth of turbulence $(W)$ is equal to earning $(E)$ of turbulence multiplied by time $(T)$ of turbulence.

$$
\underbrace{\left.\langle\rho\rangle u_{i}^{\prime} u_{j}^{\prime}\right]}_{\text {Wealth }(W)} \cong T \cdot \underbrace{\left(P_{i j}+G_{i j}\right)}_{\text {Earning }(E)}+\frac{2}{3}\langle\rho\rangle k
$$

where, $T$ means turbulent time scale, $P_{i j}$ and $G_{i j}$ mean production terms for Reynolds-stress by velocity gradient and buoyancy effect, respectively. In the case that the buoyancy effect is not considered, RANS analysis with $k-\varepsilon$ model represents the Reynolds-stress as shown in Eq. (8). Therefore, the Reynolds-stress taking account of buoyancy effect is represented as follows:

$$
\begin{gathered}
\langle\rho\rangle\left[u_{i}^{\prime} u_{j}^{\prime}\right] \cong T \cdot\left(P_{i j}+G_{i j}\right)+\frac{2}{3}\langle\rho\rangle k \\
=\left(1+\frac{G_{i j}}{P_{i j}}\right) T \cdot P_{i j}+\frac{2}{3}\langle\rho\rangle k \\
=-\left(1+\frac{G_{i j}}{P_{i j}}\right) \mu_{t}\left(\frac{\partial\left[u_{i}\right]}{\partial x_{j}}+\frac{\partial\left[u_{j}\right]}{\partial x_{i}}\right)+\frac{2}{3}\langle\rho\rangle k
\end{gathered}
$$

In Katsuki model, $G_{i j} / P_{i j}$ is approximated by $G_{k} / P_{k}$ for applying $k-\varepsilon$ modeling, $G_{k}$ means production term of turbulent kinetic energy by buoyancy effect (Eq. (22)).

$$
G_{k}=-\frac{\mu_{t}}{S_{c t}} \frac{g}{\langle\rho\rangle} \frac{\partial\langle\rho\rangle}{\partial z}
$$

where, $P_{k} / G_{k}$ corresponded to the gradient Richardson number $\left(R_{i}\right)$ multiplied by -1 . Therefore, the eddy viscosity coefficient in case with Katsuki model is derived by Eq. (23):

$$
\mu_{t}=C_{\mu} f_{\mu}\left(1-R_{i}\right) \frac{k^{2}}{\varepsilon}
$$

In addition, threshold value of $R_{i}$ is set for calculation stability as follows:

$$
0.01 \leq 1-R_{i} \leq 1.99
$$

\subsection{Numerical Discretization and Algorithm}

The numerical simulations are performed with the OpenFOAM code, which is an open source CFD code developed by the OpenFOAM ${ }^{\circledR}$ Foundation [11]. The second-order accurate central difference scheme was applied to the spatial discretization, and the PISO (pressure-implicit with splitting operaters) method [12] was used as numerical algorism to solve the governing equations. Euler implicit method is used as the time marching method.

\section{Analyses Model and Condition}

Fig. 1 shows a numerical model for the density stratification layer of multicomponent gas affected by buoyant jet in a small vessel. We set the length, width and height of the test vessel to $1.5 \mathrm{~m}, 1.5 \mathrm{~m}$ and $1.8 \mathrm{~m}$, respectively. The nozzle of diameter $0.03 \mathrm{~m}$ and height $0.1 \mathrm{~m}$ is placed at the radial center in the bottom plane of the test vessel for injecting vertical buoyant jet. Four venting nozzles, each of $0.03 \mathrm{~m}$ in diameter, are set at the bottom of the vessel. In density stratification layer, two zones are defined as shown in Fig. 1: gradient zone and reservoir zone. The helium mass fraction gradually increases in the upward direction in the gradient zone, and the mass fraction in the reservoir zone is constant.

Table 1 shows the detailed boundary and initial conditions for both LES and RANS analyses. Average inlet velocity is set to $1.5 \mathrm{~m} / \mathrm{s}$, and the radial profile is assumed to be uniform. The inlet mass gas fraction of air and helium are 0.978 and 0.022 , respectively. In addition, we use the inlet turbulence condition of $1.5 \%$ of the average flow for the $k$ and $\varepsilon$ in RANS analyses and fluctuation in LES analyses, supposing that, the inlet velocity is steady and the turbulence intensity is very small. The turbulences Schmidt number $\left(S c_{t}\right)$ and Prandtl number $\left(P r_{t}\right)$ are set to 1.0 as 


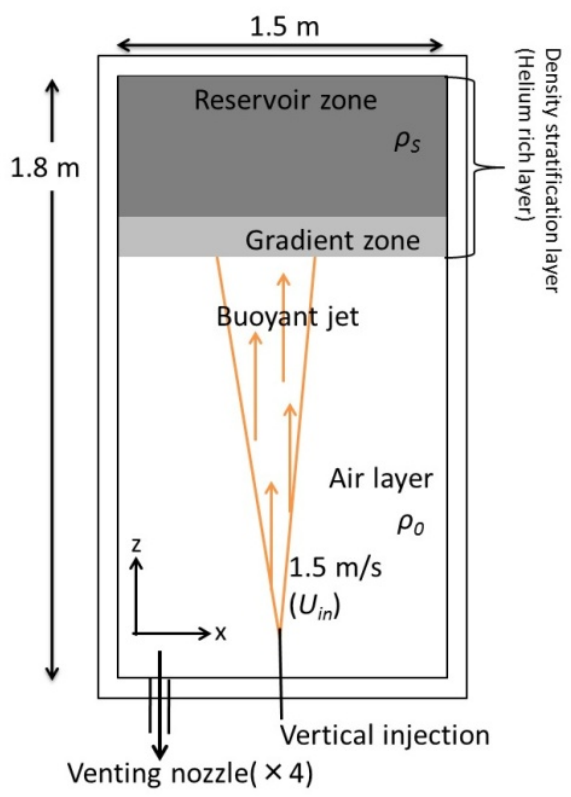

Fig. 1 Numerical analysis model for density stratification layer with vertical jet in a small vessel.

Table 1 Boundary and initial conditions.

\begin{tabular}{lll}
\hline \multirow{3}{*}{ Inlet } & Velocity & $1.5 \mathrm{~m} / \mathrm{s}$ \\
& Mass fraction & He: 0.022 \\
& Pressure & Air: 0.978 \\
& Temperature & $293.15 \mathrm{~K}$ \\
\hline \multirow{4}{*}{ Outlet } & Velocity & Advective \\
& Mass fraction & Gradient zero \\
& Pressure & $1,013 \mathrm{hPa}$ \\
\hline \multirow{4}{*}{ Wall } & Temperature & Gradient zero \\
& Velocity & No-slip \\
& Mass fraction & Gradient zero \\
& Pressure & Gradient zero \\
& Temperature & Gradient zero \\
\hline \multirow{3}{*}{ Initial condition } & Mass fraction & Fig. 2 \\
& Pressure & $1,013 \mathrm{hPa}$ \\
& Temperature & $293.15 \mathrm{~K}$ \\
\hline
\end{tabular}

the base analysis case. In LES analysis, the Smagorinsky constant $\left(C_{S G S}\right)$, SGS Schmidt number $\left(S c_{S G S}\right)$ and Prandtl number $\left(P r_{S G S}\right)$ are set to 0.158 , 1.0 and 1.0 , respectively.

Fig. 2 shows the initial vertical distribution of helium mass fraction. The distribution is determined by reference to the PANDA experimental series in SETH-2 project [2]. The cell numbers for the LES and RANS analyses are about 3 million and 0.57 million, respectively.

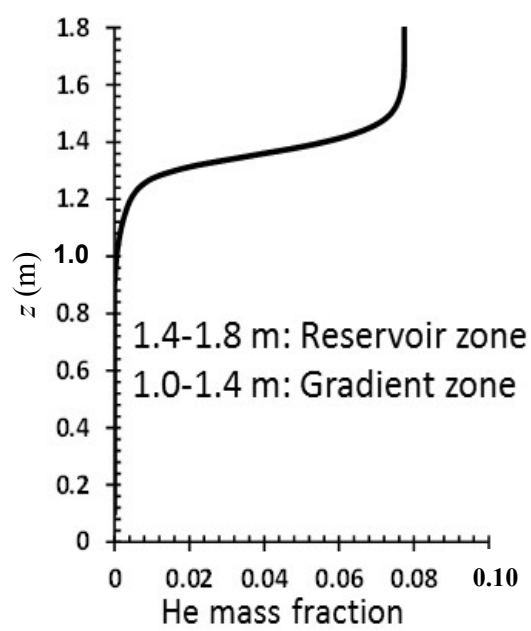

Fig. 2 Initial vertical distribution of helium mass fraction.

\section{Analyses Results and Discussion}

\subsection{Base Analyses Results}

Figs. 3 and 4 show instantaneous helium mass fraction fields and velocity vectors in the small vessel at $10 \mathrm{~s}$ and $60 \mathrm{~s}$, respectively. It is clearly shown that, the density stratification in each analysis case is gradually eroded by turbulent mixing which is produced by jet penetration into the density stratification. The erosion rate in RANS analysis without the model modification is clearly faster than that of LES analyses. The result suggested that, the

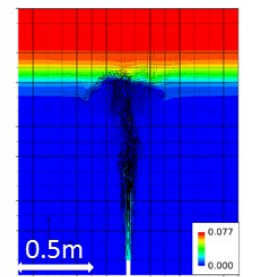

(a) LES

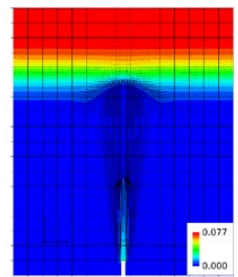

(b) No revised RANS

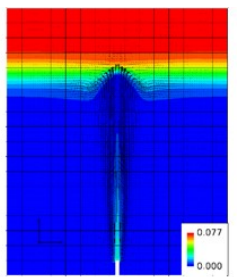

(c) Revised RANS
Fig. 3 Instantaneous helium mass fraction and velocity vectors at $10 \mathrm{~s}$.

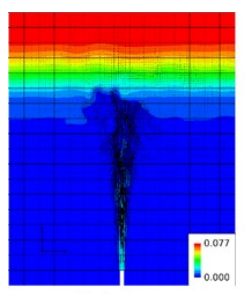

(a) LES

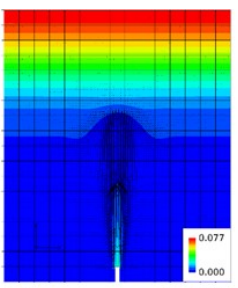

(b) No revised RANS

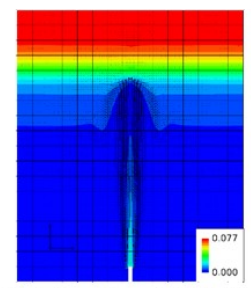

(c) Revised RANS
Fig. 4 Instantaneous helium mass fraction and velocity vectors at $60 \mathrm{~s}$. 

Gas by Buoyant Jet in a Containment Vessel

RANS analysis may overestimate the turbulence mixing in the density stratification as discussed in the previous study [1]. Meanwhile, the erosion rate in the case with the modified $k$ - $\varepsilon$ model seems to be similar to the LES results.

It can be observed from the numerical results that, the vertical buoyant jet penetrates into the gradient zone, the stagnation point is observed at about $1.3 \mathrm{~m}$ height form injection, and the jet rebounds due to negative buoyancy, which means that the heavy fluid falls back.

These jet behaviors are qualitatively the same as that observed in the previous study [13]. However, there is a clear difference between the velocity field of the LES and RANS analysis without the turbulence model modification. Fig. 5 compares the radial distribution of the average vertical velocities at the elevation of $0.9 \mathrm{~m}$ just below the gradient zone. These distributions are normalized by injection diameter $(D)$ and velocity $\left(\left[u_{i n j}\right]\right)$. In case without the turbulence model modification, the spread of vertical jet is larger, and the peak velocity at the center point is lower than that of the LES analysis. Meanwhile, in case with the modified $k-\varepsilon$ model, the velocity distribution is related more closely to the LES analysis results. It suggests that, the modified model may also improve the estimation for the velocity field.

Fig. 6 shows radial distributions of turbulent helium-mass vertical fluxes at $10 \mathrm{~s}$ at the interface elevation of $1.3 \mathrm{~m}$ where the stagnation point is located. The vertical fluxes in each analyses are normalized by injection flux $\left(F_{H e \_i n j}\right)$ calculated from injection velocity, density and helium mass fraction. The negative value of the vertical flux indicates the magnitude of mixing due to the turbulence, while the positive value indicates separation. The LES analysis result indicates that, the negative vertical flux occurs near the center of the jet $(r / D<4)$, while, the vertical flux at the outside region $(r / D>6)$ is almost zero, indicating no vertical mixing. It is noteworthy that, the vertical flux becomes positive where the mixture

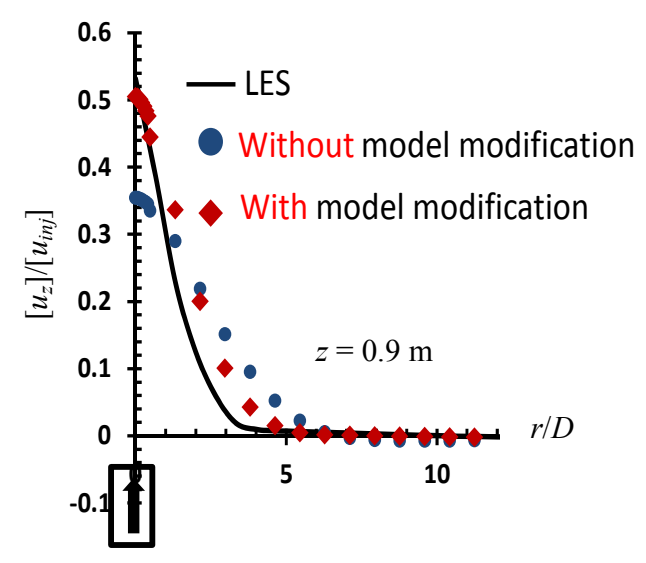

Jet injection nozzle

Fig. 5 Radial profile of mean vertical velocity.

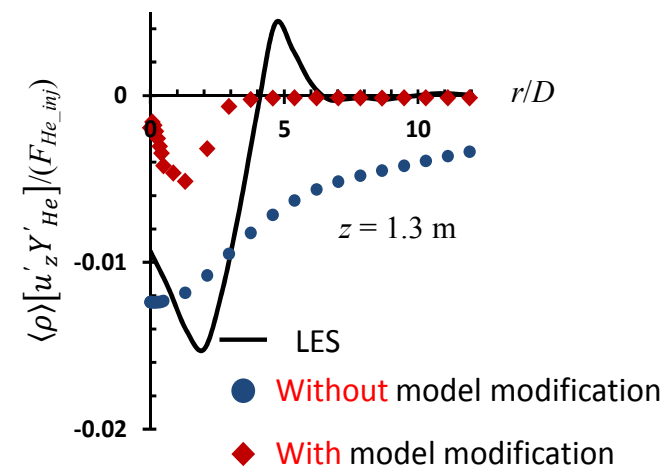

Fig. 6 Radial profile of turbulent helium-mass vertical flux at $10 \mathrm{~s}$.

separation occurs due to the jet rebounding as faintly shown in Fig. 3. This behavior is called "the counter-gradient diffusion" [14]. The vertical flux in case without the turbulence model modification becomes negative value in all radial location. It means that, the vertical turbulence mixing occurs in a wider range. This is supposed to be the reason for the overestimation of the density stratification layer erosion by the RANS analyses discussed in the previous study [1]. Meanwhile, in case with the modified $k-\varepsilon$ model, the radial distribution profile is in qualitative agreement with the LES analyses result. The decrease in negative flux in the center region $(r / D$ $<2$ ) can be simulated owing to applying the Kato-Launder modification [7] for suppressing the excessive estimation of turbulent kinetic energy production around the stagnation point. The vertical flux at outside region $(r / D>6)$ becomes almost zero 
owing to applying Katsuki model [8] for considering the turbulence damping in the density stratification layer. The counter-gradient between 4 and 6 of $r / D$ cannot be simulated by the both RANS analyses because of using gradient diffusion hypothesis for the turbulent scalar flux modeling.

The above results suggest that, the turbulence mixing mechanism can be simulated better with the modified turbulence model compared to the low-Re type $k-\varepsilon$ model, although the calculated magnitude of the turbulent mixing in the center region is considerably smaller than that of LES analysis. This suggests a need for the parametric study about the turbulence Schmidt number $\left(S c_{t}\right)$ and so on. The following shows a brief parametric study about $S c_{t}$.

\subsection{Parametric Study about Turbulent Schmidt Number}

While, the helium transport mechanism in the center region may be qualitatively simulated by the modified $k-\varepsilon$ model as shown above, the analysis result suggests necessity for the parametric study about the turbulence Schmidt number $\left(S c_{t}\right)$ and so on. The following shows a brief parametric study about $S c_{t}$. The $S c_{t}$ is set to 0.5 and 0.3 for parametric analyses as trial values.

Figs. 7 and 8 show instantaneous helium mass fraction fields and velocity vectors in each parametric analysis at $10 \mathrm{~s}$ and $60 \mathrm{~s}$, respectively. Fig. 9 shows the radial distribution of the normalized average vertical velocities at the elevation of $0.9 \mathrm{~m}$. There is no obvious difference of both helium mass fraction distributions and velocity fields among the base and parametric analyses. Fig. 10 shows radial distributions of normalized turbulent helium-mass vertical fluxes at $1.3 \mathrm{~m}$. The distribution gradually gets close to the LES analysis results with decreasing $S c_{t}$.

Although both the LES and RANS analyses require further validation with the experiment data as a future work, the approach for modifying turbulent model is beneficial in the improvement of analysis for density stratification layer behavior in containment vessel. As an additional future work, the comparisons with large-scale experiments are required for the capability assessment of the turbulence model modification.

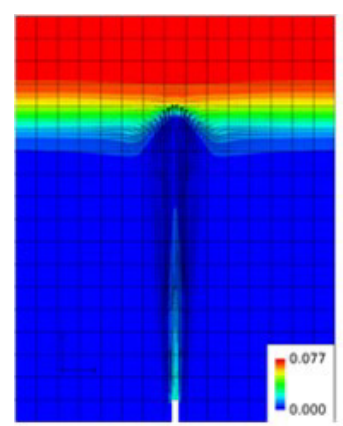

(a) Revised RANS $\left(S c_{t}=0.5\right)$

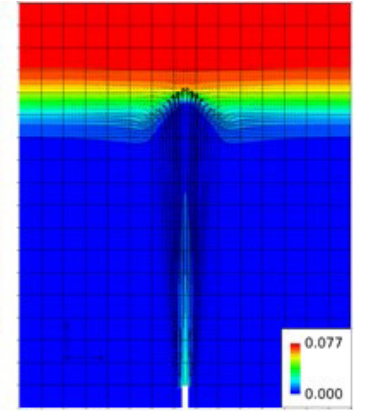

(a) Revised RANS $\left(S c_{t}=0.3\right)$
Fig. 7 Instantaneous helium mass fraction and velocity vectors at $10 \mathrm{~s}\left(\mathrm{Sc}_{t}=\mathbf{0 . 5}\right.$ and 0.3$)$.

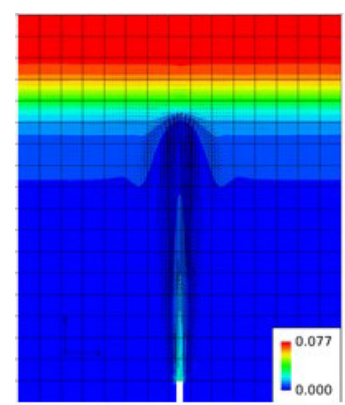

(a) Revised RANS $\left(S c_{t}=0.5\right)$

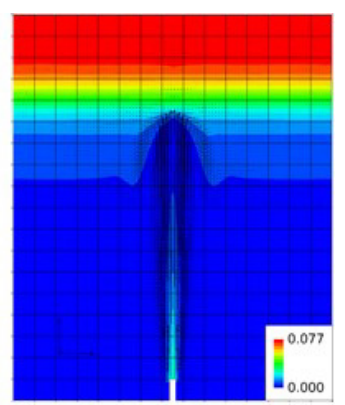

(a) Revised RANS $\left(S c_{t}=0.3\right)$
Fig. 8 Instantaneous helium mass fraction and velocity vectors at $60 \mathrm{~s}\left(S c_{t}=0.5\right.$ and 0.3$)$.

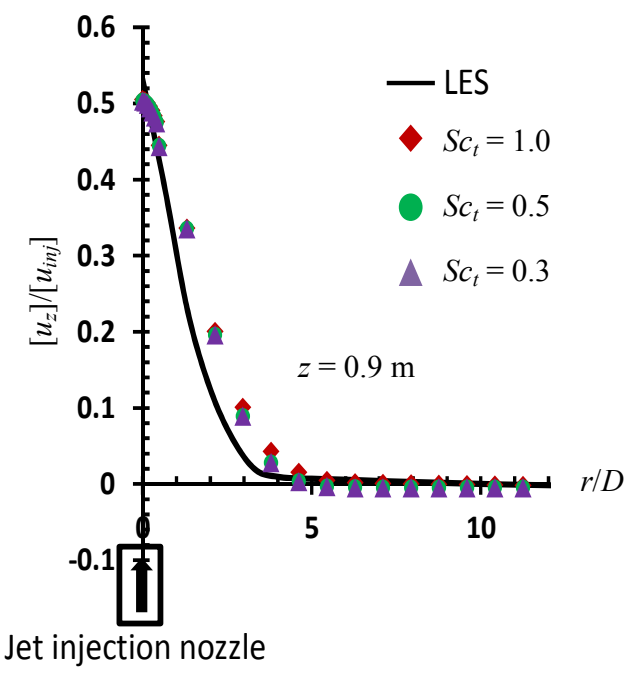

Fig. 9 Radial profile of mean vertical velocity $\left(S c_{t}=1.0,0.5\right.$ and 0.3 ). 


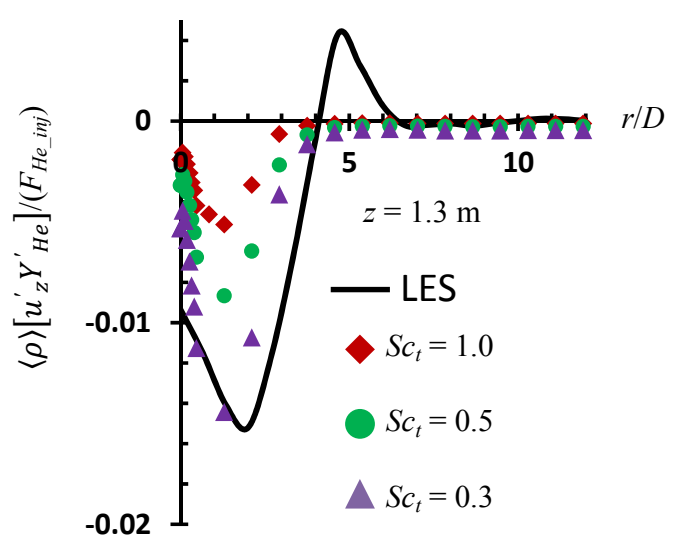

Fig. 10 Radial profile of turbulent helium-mass vertical flux at $10 \mathrm{~s}\left(S c_{t}=1.0,0.5\right.$ and 0.3$)$.

\section{Conclusions}

RANS and LES analyses were performed to simulate density stratification behavior affected by upward vertical buoyant jet discharged from the bottom in a small vessel. Two types of turbulent models are applied for the RANS analyses. The first is the low Reynolds number $k-\varepsilon$ type developed by Launder and Sharma. The second is revised from the first model in order to accurately predict turbulent production and damping around density stratification layer.

The vertical jet penetration into the density stratification was observed clearly in all analyses cases. The vertical velocity profile in case with the second $k-\varepsilon$ model was better agreement with the LES analysis data than that of the first $k-\varepsilon$ model. The erosion of the density stratification in case with the first $k$ - $\varepsilon$ model was faster than other analyses cases. The erosion rate simulated with the second $k-\varepsilon$ model seemed to be similar to that of LES analysis result. Turbulent helium vertical flux profile in case with LES analysis suggested that, the turbulence mixing occurred only near the center of the jet, and the counter gradient diffusion occurred in the jet rebounding region. The turbulence mixing only near the center of the jet could be simulated only with the second $k-\varepsilon$ model in the RANS analyses, while the counter gradient diffusion could not simulate by the both RANS analyses because of using gradient diffusion hypothesis. The size of the overall profile could be revised by turbulent Schmidt number parametric adjustment. Although more detailed validations of both the LES and RANS analyses based on appropriate experimental data are required as a future work, the applied modification turbulence model has a good potential to improve the precision of density stratification behavior in a containment vessel.

\section{References}

[1] Allelein, H. J., Fischer, K., Vendel, J., Studer, E., Schwarz, S., Houkema, M., Paillère, H., and Bentaib, A. 2007. International Standard Problem ISP-47 on Containment Thermal-Hydraulics. Final report.

[2] Paladino, D., Mignot, G., Kapulla, R., Zboray, R., Andreani, M., Tkatschenko, I., Studer, E., and Brinster, J. 2013. "OECD/SETH-2 Project: PANDA and MISTRA Experiments Addressing Key Safety Issues for Water Reactor Containment." Presented at the 15th International Topical Meeting on Nuclear Reactor Thermal-Hydraulics, Pisa, Italy.

[3] Andreani, M., Kapulla, R., and Zboray, R. 2012. "Gas Stratification Break-Up by a Vertical Jet: Simulations Using the GOTHIC Code." Nuclear Engineering and Design 249 (August): 71-81.

[4] Yonomoto, T., Sibamoto, Y., Ishigaki, M., and Abe, S. 2015. "The ROSA-SA Project on Containment Thermal Hydraulics." Presented at the International Experts Meeting on Strengthening Research and Development Effectiveness in the Light of the Accident at the Fukushima Daiichi Nuclear Power Plant, Vienna, Austria.

[5] Launder, B. E., and Spalding, D. B. 1974. "The Numerical Computational of Turbulent Flows." Computer Methods in Applied Mechanics and Engineering 3 (2): 269-89.

[6] Launder, B. E., and Sharma, B. I. 1974. "Application of the Energy-Dissipation Model of Turbulence to the Calculation of Floe Near a Spinning Disc." Letters in Heat and Mass Transfer 1 (2): 131-8.

[7] Kato, M., and Launder, B. E. 1993. "The Modeling of Turbulent Flow around Stationary and Vibrating Square Cylinders." In Proceedings of the 9th Symposium on Turbulent Shear Flows, 10.4.1-10.4.6.

[8] Katuki, T., Ooka, R., Takahashi, T., and Kato, S. 2009. "Wind Tunnel Experiment and Numerical Simulation of Atmosphere Boundary Layer under Various Atmospheric Stability." Journal of Environmental Engineering 74 (640): 735-43.

[9] Smagorinsky, J. 1964. "General Circulation Experiments with the Primitive Equations, I. the Basic Experiment." 


\section{Gas by Buoyant Jet in a Containment Vessel}

Monthly Weather Review 91 (3): 99-164.

[10] Launder, B. E. 1988. "On the Computation of Convective Heat Transfer in Complex Turbulent Flows.” Journal of Heat Transfer 110 (November): 1112-28.

[11] OpenCFD Ltd. 2012. "The Open Source CFD Toolbox User's Guide.” OpenCFD Ltd. Accessed June 01, 2015. http://cfd.direct/openfoam/user-guide.

[12] Issa, R. 1986. "Solution of the Implicity Discretized Fluid Flow Equations by Operating-Splitting." Journal of Computational Physics 62 (1): 40-65.
[13] Studer, E., Brinster, J., Tkatschnko, I., Mignot, G., Paladino, D., and Andreani, M. 2012. "Interaction of a Light Gas Stratification Layer with an Air Jet Coming from below: Large Scale Experiments and Scaling Issues." Nuclear Engineering and Design 253 (December): 406-12.

[14] Komori, S., and Nagata, K. 1996. "Effect of Molecular Diffusivities on Counter-Gradient Scalar and Momentum Transfer in Strongly Stable Stratification." Journal of Fluid Mechanics 326 (November): 205-37. 